\title{
Personal Navigation via Shoe Mounted Inertial Measurement Units
}

\author{
Ozkan Bebek, Michael A. Suster, Srihari Rajgopal, Michael J. Fu, Xuemei Huang, M. Cenk Cavusoglu, \\ Darrin J. Young, Mehran Mehregany, Antonie J. (Ton) van den Bogert, and Carlos H. Mastrangelo
}

\begin{abstract}
We are developing a personal micronavigation system that uses high-resolution gait-corrected inertial measurement units. The goal of this project is to develop a navigation system that use secondary inertial variables, such as velocity, to enable long-term precise navigation in the absence of Global Positioning System (GPS) and beacon signals. In this scheme, measured zero velocity durations from the ground reaction sensors are used to reset the accumulated integration errors from the accelerometers and gyroscopes in position calculation. We achieved an average position error of 4 meters at the end of half-hour walks.
\end{abstract}

\section{INTRODUCTION}

We are developing a personal micronavigation system that uses high-resolution gait-corrected inertial measurement units. The system combines a commercial off-theshelf (COTS) inertial measurement unit (IMU) with a high-resolution, thin, flexible, error-correcting biomechanical ground reaction sensor cluster (GRSC) connected to a handheld processing and read-out unit. The final sensor parts including the IMU and the GRSC will be placed within the heel and at the sole of a personnel boot and wirelessly connected to a handheld unit which will process the data in real-time. In this approach the IMU will measure inertial displacements, and the GRSC will independently measure dynamic ground forces, shear strains and sole deformation associated with ground locomotion gait. The high resolution biomechanical GRSC data can be used to detect periods of zero velocity accurately. These zero velocity points provide discrete zero velocity corrections to the IMU that dramatically increase its effective positioning resolution.

Step corrected (also known as dead reckoning) IMU and GPS navigation systems have been in existence for several years [1]-[10], but unlike our proposed approach these

This work was supported in part by the Micro Inertial Navigation Technology (MINT) program of the Defense Advanced Research Projects Agency (DARPA). The views, opinions, and/or findings contained in this article/presentation are those of the author/presenter and should not be interpreted as representing the official views or policies, either expressed or implied, of the Defense Advanced Research Projects Agency or the Department of Defense.

O. Bebek, M. A. Suster, S. Rajgopal, M. J. Fu, M. C. Cavusoglu, and M. Mehregany are with the Department of Electrical Engineering and Computer Science, Case Western Reserve University, Cleveland, Ohio, 44106 , USA. oxb6@case.edu, sxr61@case.edu, mas20@case.edu, mjf24@case.edu, cavusoglulcase.edu, mehran@case.edu

A. J. van den Bogert, and Xuemei Huang are with the Department of Biomedical Engineering of Lerner Research Institute, Cleveland Clinic, Cleveland, Ohio, 44195, USA. bogert@bme.ri.ccf.org, huangx2@ecf.org

D. J. Young, and C. Mastrangelo are with the Department of Electrical and Computer Engineering, University of Utah, Salt Lake City, Utah, 84112, USA. darrin.youngeutah.edu, mastrang@ece.utah.edu

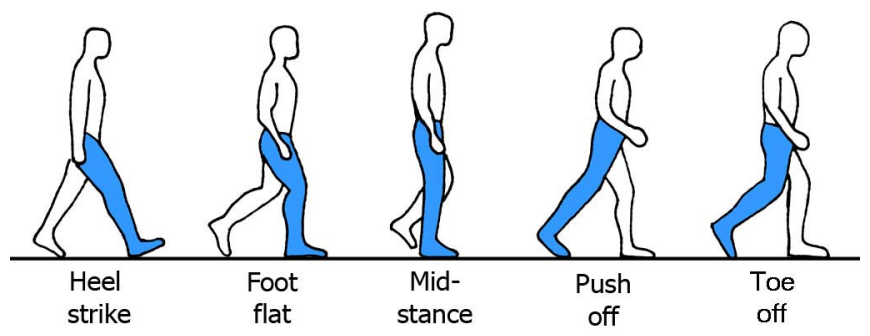

Fig. 1. Stance phase in human bipedal locomotion. In this phase the foot is in contact with the ground. Only at specific times, during midstance, the foot heel remains stationary (Figure modified from [12]).

systems detect the step impact shock with accelerometers placed away from the ground. This approximate detection technique typically results in a $1-2 \%$ positioning error. In our approach, we use a data-rich high-resolution GRSC placed very close to the point of heel to ground contact to provide detailed contact information to an IMU located in close proximity to the GRSC. We believe this extra information and the close mechanical (near rigid) relation between the velocity at the GRSC and IMU locations [11] are key to reach the high resolution positioning improvements. Our goal with this unique sensor data fusion approach is to ultimately permit accurate navigation on any indoor or outdoor terrain, unassisted by external signals.

In human bipedal locomotion, the walking mode or gait consists of two separate phases [12]. In the swing phase, the leg is off the ground. This period extends from the instant the toe leaves the ground until the heel strikes. In the stance phase (Figure 1), the foot heel first contacts the ground, then it rolls until midstance is reached resulting in pivoting of the leg on the ankle (and corresponding forward motion of the body). Beyond midstance, detachment of the foot takes place through a gradual rolling. It is evident that only during a fraction of the midstance the velocity of the heel is exactly zero [13]. Hence we propose to detect this time period very precisely with the GRSC. A high density GRSC can detect very small changes in the stationary contact yielding very small errors in the velocity determination in the stance phase. Relating the velocity of the rolling contact to the the heel velocity, where IMU is located, can provide us to detect zero velocity points.

Our initial design goals for the personal micronavigation system are: (i) navigation accuracy below 10 meters for $1 / 2$ hour walking; (ii) velocity sensing bias per step below 4 $\mathrm{mm} / \mathrm{s}$; (iii) form factor for the GRSC below $10 \mathrm{cc}$; (iv) power consumption of the GRSC below $300 \mathrm{~mW}$; and (v) GRSC 

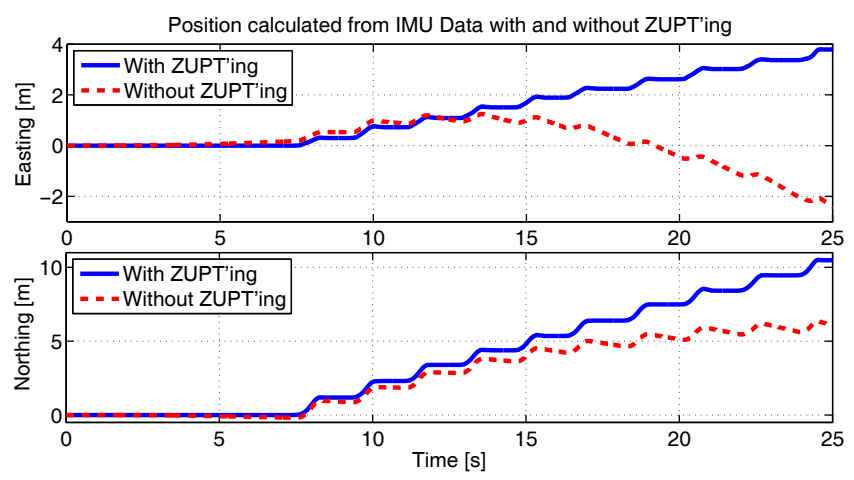

Fig. 2. Comparison of position calculations with and without zerovelocity updating (ZUPT'ing). The user walks on a straight line headed towards north-east. The figure shows the position of the user with respect to time, where Easting refers to the eastward-measured distance, and Northing refers to the northward-measured distance. Without zero-velocity correction, drifting errors build up in few seconds. Dashed lines in the figure indicate the drifted position.

with at least 10 sensor elements. If these design goals are met, the developed system will exceed the current state of the art micronavigation systems.

\section{NAVIGATION With IMU AND ZERO-VELOCITY UPDATING}

The basic idea behind a gait-corrected navigation system is to use the walking stride to periodically reset the drifting IMU, thus dramatically reducing cumulative navigation errors. The correction occurs when the foot is on the ground, when all velocities and accelerations of the shoe are zero. Without this correction, even smallest measurement errors, due to sensor drift or sensor noise, will amplify and cause drifting errors, meters in 4-5 steps. Figure 2 compares the position calculated by integrating the acceleration twice with zero-velocity updating (ZUPT'ing) during stance period, and the position calculated without any velocity updates.

The effectiveness of stance-based zero-velocity updating depends on the detection of zero velocity at the stance period. Most personal dead-reckoning systems detect steps using a pedometer or accelerometer, and move the position estimate forward by the step length in the direction determined by a magnetic compass or a yaw gyroscope [6], [14]-[16]. The sensors which are attached to the upper body, detect motion from normal acceleration or phasing acceleration axes that exhibit cycles typical of a human's walking motion. The number of steps is counted in a pedometer system. The average step length is adjusted for the walking speed, and then used to calculate the user's position. More sophisticated systems analyze the accelerometer signals to estimate step lengths. All of these systems require calibration to an individual user because everyone's gait has different acceleration profiles. An inertial navigation system embedded in a soldier's boot heel is described by Elwell [17], but no experimental validation was performed. Stirling et al. [18] describe an experiment using a prototype shoe-mounted sensor that measures stride length with accelerometers and direction with magnetometers. The system measures angular acceleration using pairs of accelerometers. The system stops integrating and resets the velocity before each step. Errors up to $20 \%$ of distance traveled were reported.

A more complex pedometer-like approach was introduced by Cho and Park [19]. Their system uses a two-axis accelerometer and a two-axis magnetometer located on a shoe. Step length is estimated from accelerometers readings that are passed through a neural network, and a Kalman filter was used to reduce magnetic disturbances. Although their outdoor results are good, they could not filter the magnetic disturbances well indoors, which resulted in large errors. A fiducial-based position estimation system was proposed by Saarinen [20]. Ultrasonic sensors attached to boots were used to measure the length of every stride in real-time. In straightline walking experiments the authors report an average and maximum error of $1.3 \%$ and $5.4 \%$, respectively. Another fiducial-based approach introduced by Brand [21] uses radio frequency (RF) phase changes between a reference signal located in a waist pack and the one coming from a transmitter located on each boot. This system's measurements are limited to $2 \mathrm{D}$ environments and cannot detect altitude changes.

Recently Ojeda and Borenstein [9], [10] developed a shoebased navigation system that uses a small 6-DOF inertial measurement unit (IMU) attached to the user's boot. The IMU provides rate-of-rotation and acceleration measurements that are used in real-time to estimate the location of the user relative to a known starting point. In order to reduce the most significant errors of this IMU-based system they used ZUPT'ing. With the ZUPT technique and related signal processing algorithms, relative error of the system was about $2 \%$ of the distance traveled. In this typical personal dead-reckoning (PDR) system, the error is independent of the gait or speed of the user. Their PDR system works in $3 \mathrm{D}$ environments, although errors in z-direction are usually larger than $2 \%$ of distance traveled. Feliz et al. used an IMU unit and a GPS and barometer unit in their PDR system [22]. They did short indoor and outdoor walks to test the system. Their best relative position loop-closing errors were around $2 \%$ for outdoor walks and $10 \%$ indoor walks.

Many of the PDR devices attempt to perform the ZUPT'ing by detection of the contact of the foot with the ground. Most stance based schemes in the literature equate zero velocity detection to the impact of the heel when it hits the ground. The problem with this scheme is that the impact shock event only signals the beginning of the stance phase which involves several sub phases itself. Not all the sub phases has zero velocity. Zero velocity only occurs at some point around the midstance subphase, after all rolling contact of the foot with the ground has been reached. If the zero velocity point is not accurately determined, the resulting ZUPT'ing scheme will have an intrinsic zero-velocity bias which will reduce its effectiveness. In order to detect zerovelocity stance accurately, its is necessary to utilize a sensor at the IMU location to record sufficient data detailing the nature of the contact with the ground. In this work, we propose to use a GRSC placed in close proximity to the 


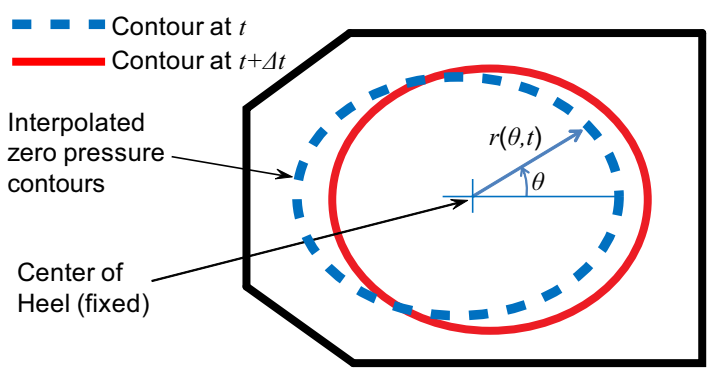

Fig. 3. Propagation of a pressure contour created by the heel.

IMU location to more accurately estimate the periods of zero velocity, improving ZUPT'ing and reducing position error.

\section{Inertial MEASUREMENT Unit (IMU)}

We used an InterSense InertiaCube 3 module that integrates a two two-axes accelerometers, three single-axis gyroscopes and a three-axes magnetometer compass within a low volume $\left(26.2 \times 39.2 \times 14.8 \mathrm{~mm}^{3}\right)$, which can fit within the heel. This IMU module is temperature compensated producing a lower error bias than other COTS IMU units. InterSense [23] has developed one of the most sophisticated extended Kalman filter schemes for micronavigation. InertiaCube 3 combines the aforementioned sensing elements with an integrated Kalman filtering algorithm. The unit can provide orientation and gravity compensated acceleration information aligned with earth's magnetic north. InertiaCube3 can measure accelerations up to $\pm 6 \mathrm{~g}$.

\section{Pressure SENSOR}

In our walk experiments, we used a COTS pressure sensor array, DigiTacts II Array Tactile sensors manufactured by Pressure Profile Systems (PPS), as our biomechanical GRSC sensor. DigiTacts II sensors have 24 sensing elements with sensing area of $82.3 \times 45.1 \mathrm{~mm}^{2}$. These sensors use a capacitance-based sensing scheme that can detect pressures up to $140 \mathrm{kPa}$. Overall power consumption of the GRSC, was $20 \mathrm{~mW}(3.3 \mathrm{~V} / 6 \mathrm{~mA})$. The GRSC was a custom built unit that was calibrated prior to shipment.

Pressure sensor arrays can be used to detect pressure contours that are generated by the heel if the sensor is placed between the heel of the shoe and the shoe insole. One can find the centroid velocity of the pressure contours (Figure 3 ) as

$$
\begin{aligned}
\vec{v}(\theta, t)=\frac{d r(\theta, t)}{d t} \cdot \widehat{r} & \approx \frac{r(\theta, t+\Delta t)-r(\theta, t)}{\Delta t} \cdot \widehat{r}, \\
\vec{v}_{c n t r} & =\int_{0}^{2 \pi} \vec{v}(\theta, t) d \theta
\end{aligned}
$$

where $\vec{v}$ is the velocity of a point on the contour, $r$ is the radius of the contour point, $\widehat{r}$ is the unit vector that lies on the line between the center of the contour and the contour point, and $\vec{v}_{c n t r}$ is centroid velocity of the contour. The contour velocity can be used to detect the zero velocity of the shoe.
We can assume that the shoe, and the IMU, is at rest when the centroid velocity is below a velocity threshold

$$
\left\|\vec{v}_{c n t r}\right\| \leq v_{t h r s h}
$$

This zero velocity information can be used in the ZUPT'ing scheme to reduce the drift in the IMU measurements.

In order to verify that pressure sensor arrays can be used to detect zero-velocities of the heel, we conducted experiments using an external sensor in addition to the IMU and biomechanical sensor. A 3D optical motion capture system system, Eagle Digital RealTime System from Motion Analysis Corp. [24], was used as the external sensor. This sensor can provide 6-DOF position and orientation information with sub-millimeter RMS accuracy using optical markers. Optical markers and IMU were placed on a plate that was attached to the heel of a combat boot (Figure 4). An insole-shaped pressure sensor, Novel Pedar Pressure Sensor System [25], was placed in the boot as the biomechanical sensor. This sensor has 99 sensing elements. We used 54 of the 99 elements located in the heel portion of the sensor (Figure 5). Using all these sensors, walk data from each of the sensors were captured and calculated velocity outputs were compared. Figure 6 shows the velocity measurements from the three sensors: Boot's heel velocity along the walking direction, $v_{x}$, from optical motion capture system, the angular velocity in the pitch ${ }^{1}$ rotation, $\omega_{\text {pitch }}$, from the IMU, and the pressure contour centroid velocity of the heel, $v_{c n t r}$, from the pressure system. This figure shows that the minimum detectable velocity of the boot can be observed from the pressure contour velocity of the heel, $v_{c n t r}$. This provides additional and more accurate zero velocity detection, independent of the measurements from the IMU. The optical sensor was not used in the calibration of the overall system, which is described in the later parts of the presented work.

\section{Position Calculation From the SEnsors}

The sensor data fusion scheme that we followed to integrate acceleration is given in Figure 7. First, acceleration and orientation information, which are represented in the navigation coordinate frame, was retrieved from IMU. Navigation coordinate frame (also known as spatial coordinate frame, or world coordinate frame) is the name that was used by the IMU manufacturer, which is the locally-level geographic frame with its $x$-axis pointing north, $y$-axis east, and $z$-axis down [26]. Next, a Kalman filter (KF) [27] is employed to find acceleration biases. This estimation is performed in the sensor's body coordinate frame and then the resultant biases are transformed to the world coordinate frame. Bias compensated acceleration from the IMU and zero velocity points from the pressure sensor array are used in the integration via ZUPT'ing to calculate the position of the user. Finally, a calibration is applied to correct the drifts in the calculated position. This calibration is used to correct future collected walk data.

\footnotetext{
${ }^{1}$ Pitch is the measure of the rotation to which boot's nose tilts up or down relative to its heel.
} 


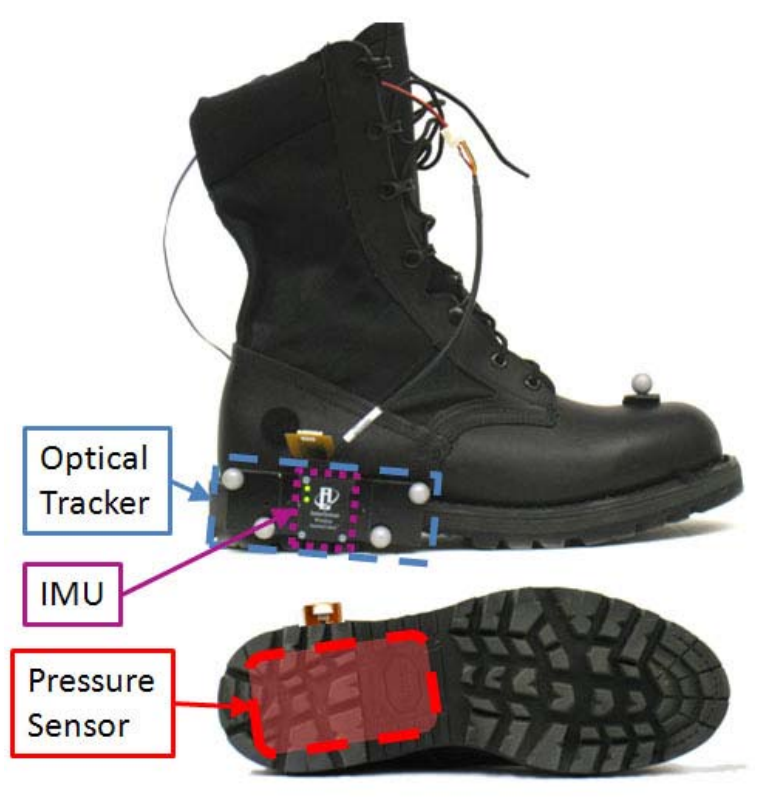

Fig. 4. Boot sensors: Boot with the pressure sensor inserted in the insole is shown. The IMU is located externally on the heel attached to an optical marker tool frame. Position and orientation of the IMU is captured using the 3D optical motion tracker system.

(2) Pressure Contour $[\mathrm{kPa}]$
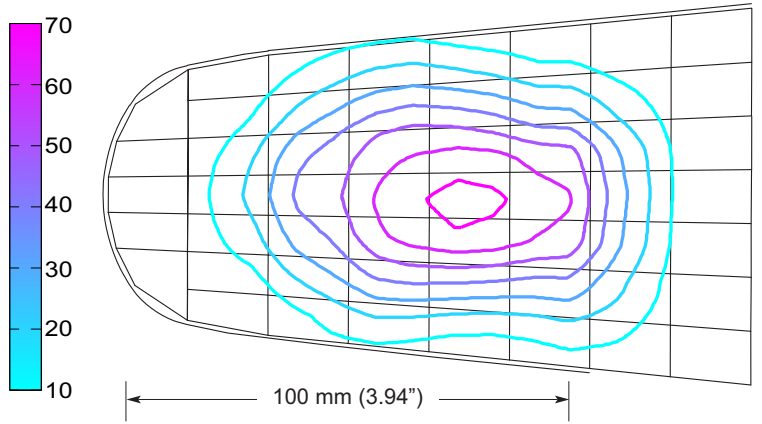

Fig. 5. Pressure contours overlayed on the heel portion of the pressure sensor insole.

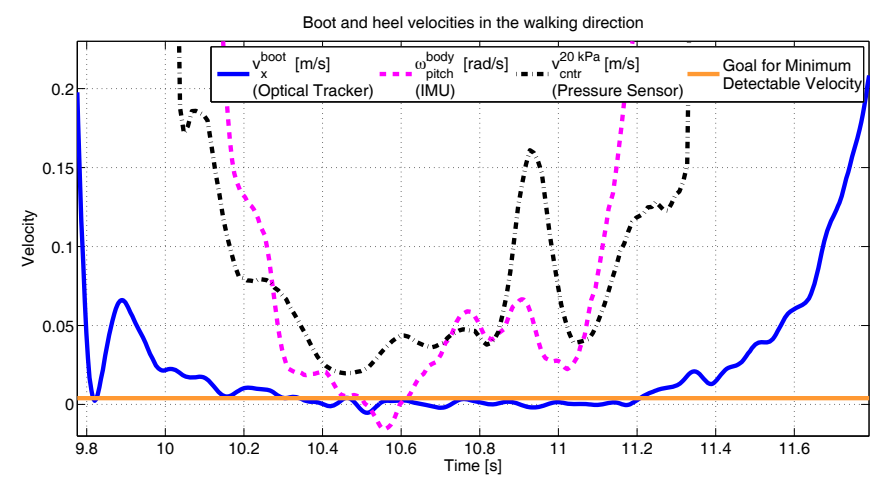

Fig. 6. Velocity measurements from optical motion capture system, IMU, and pressure sensor array during a stance period. Minimum detectable velocity sensing bias goal is $4 \mathrm{~mm} / \mathrm{s}$.

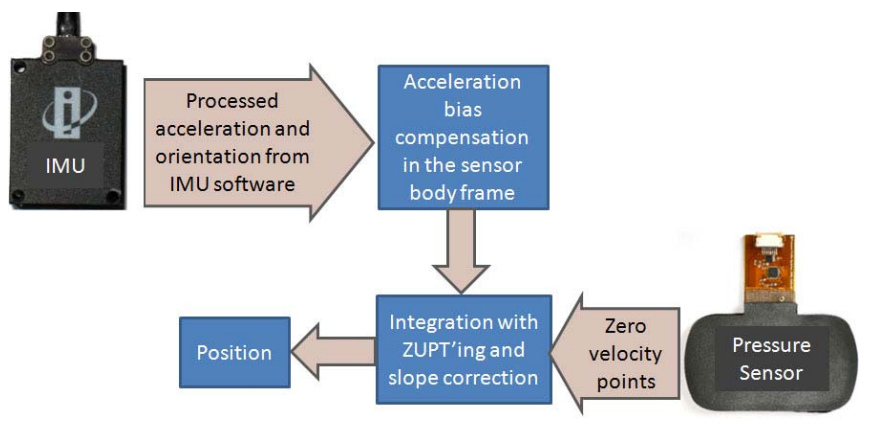

Fig. 7. Integration with ZUPT'ing scheme followed to calculate position from IMU and pressure sensor outputs.
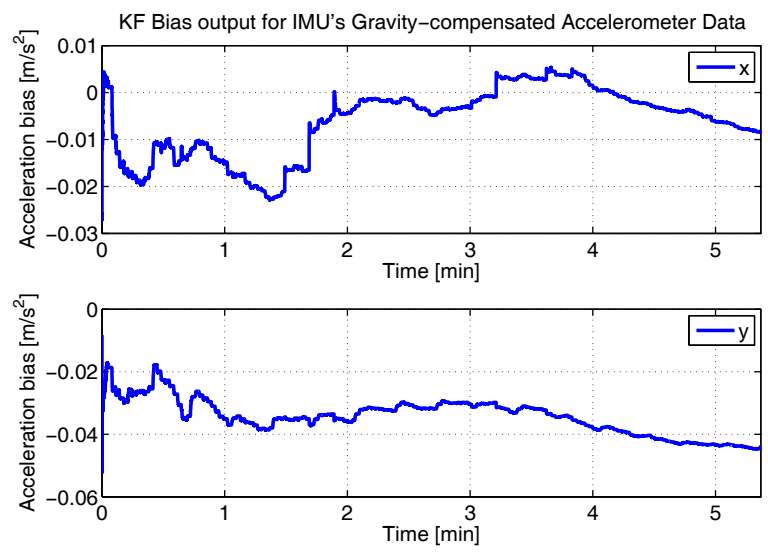

Fig. 8. Kalman filter bias output for IMU's acceleration outputs.

In the following sections the steps of this position calculation process are explained in detail.

\section{A. Acceleration bias compensation}

The IMU software provides gravity-compensated acceleration in the navigation coordinate frame, $a^{\text {nav }}$, and the matrix that defines rotation between navigation and body coordinate frames, $\mathbf{R}_{n b}$. Gravity-compensated acceleration in the sensor body coordinates, $a^{\text {body }}$, can be calculated as

$$
a^{b o d y}[k]=\mathbf{R}_{n b}^{-1}[k] a^{n a v}[k] .
$$

We fine tuned the IMU measurements via a bias estimator. IMU's acceleration output was processed with a Kalman filter to calculate the actual acceleration as

$$
a_{a}^{n a v}[k]=a_{m}^{n a v}[k]-\mathbf{R}_{n b}[k] a_{b}^{b o d y}[k]
$$

where $a_{m}^{n a v}$ is the measured acceleration in the navigation frame, $a_{b}^{b o d y}$ is the acceleration bias detected by the Kalman filter in the body frame, and $a_{a}^{n a v}$ is the actual acceleration represented in the navigation frame that exists on the heel. The bias estimation, $a_{b}^{b o d y}$, is performed in the IMU's body coordinate frame, and then subtracted from the measured acceleration in order to get the the actual acceleration in the navigation frame.

The output of the KF is plotted in Figure 8. With this extra level of filtering, the final position error was decreased about $5-10 \%$ in most of our tests. Positions calculated from 
Position Calculated from IMU's Gravity-Compensated Data (w/ZUPT'ing)

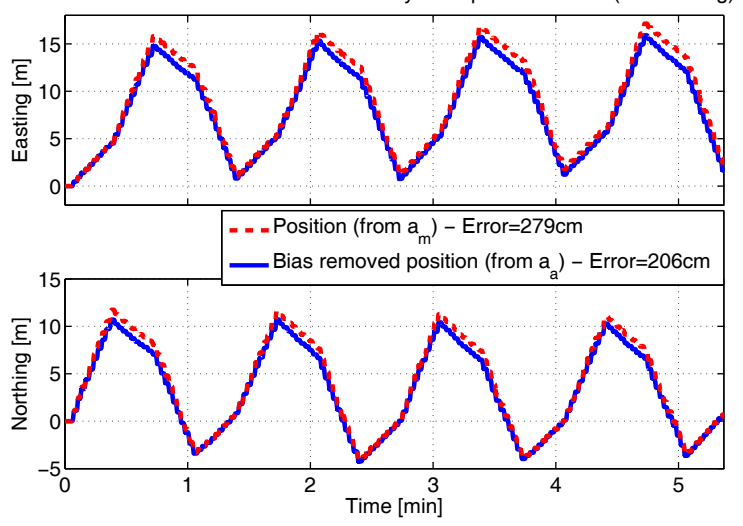

Fig. 9. Comparison of two positions calculated from measured and actual accelerations using ZUPT'ing scheme. Easting refers to the movement towards the east, and Northing refers to the movement towards the north.

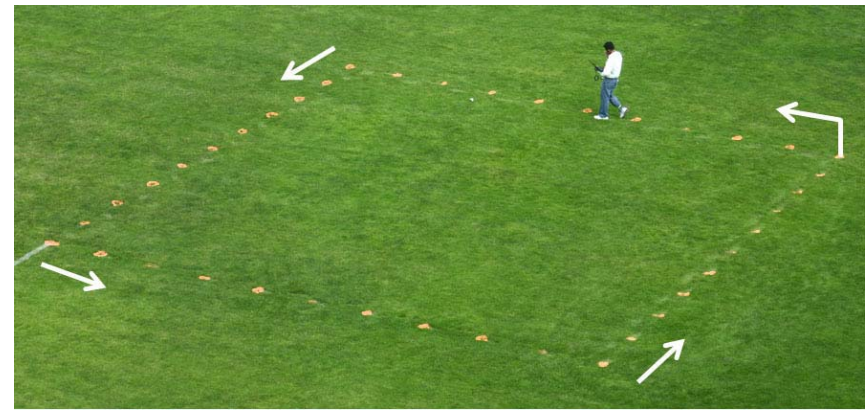

Fig. 10. Calibration step points around the square calibration loop.

measured and actual (bias compensated) accelerations are compared in Figure 9. For the case shown in this figure, the loop-closing error was decreased by $26 \%$.

\section{B. Calibration}

In initial loop-closing tests, we realized that our position results have drifts towards a fixed direction. We believe that this is due to an error in the IMU's internal gravity compensation algorithm. In order to cancel this random drift effect, we developed a calibration scheme that was applied each time we performed an experiment. We marked 40 points around a $11.7 \mathrm{~m} /$ edge square in the field such that the user can walk on these marked step points. Walking with uniform steps, the user completed 4 to 6 laps (Figure 10) around the square. Using this initial data, 40 lines were fitted through the position of the same step points (Figure 11). An average slope was determined from the linear fits, and the slope was used to correct the deviation in the future collected data.

\section{Walk Experiments}

We performed loop-closing $1 / 2$ hour walks to test our system. The procedure that we followed is depicted in Figure 12. First, we performed a 5-minute short walk to be used in the calibration process described in the previous section. Next, we performed, longer, $1 / 2$ hour walks ending at the starting point to calculate the loop-closing error for each walk.

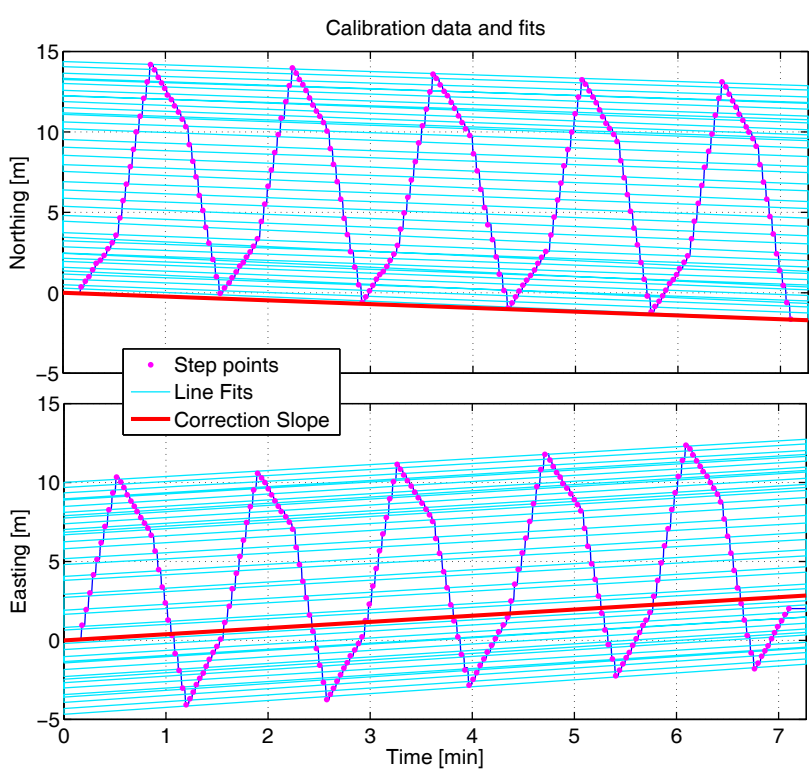

Fig. 11. Linear fits are shown for the steps during calibration walk. Average slope of the linear fits was used to correct the deviation in the future data. Easting refers to the movement towards the east, and Northing refers to the movement towards the north.

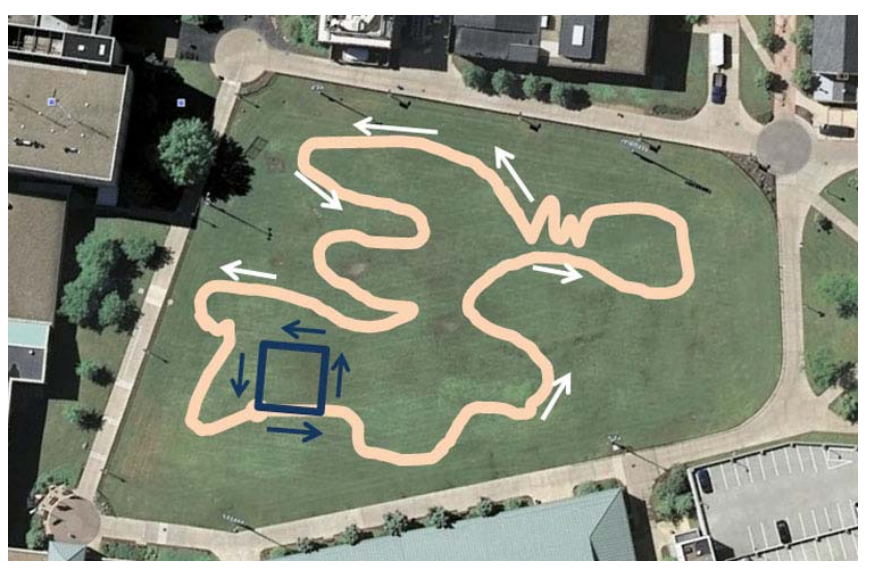

Fig. 12. Procedure for loop-closing $1 / 2$ hour walks: 1. Short walk around a square $(11.7 \mathrm{~m} /$ edge) for 5 minutes. 2. Longer, $1 / 2$ hour walks around the same square as short walk or random path (paths are shown for illustrative purposes only).

The field where we performed our walk tests is a sports field with reasonably flat surface, and we are assuming that the person is walking on a flat surface. Therefore the position errors reported in this paper are the Euclidean norm of the two-dimensional errors. We have conducted 6 half-hour loop-closing experiments; the subject first walked along the square-shaped path for about five minutes for the calibration. Then we collected additional $1 / 2$ hour walk data either around the same square as the calibration walk or random path in the field.

\section{Walk Results}

Figures 13 and 14 shows two of the walk results. "Position Error" is calculated by integrating the measured acceleration, $a_{m}$, with ZUPT'ing, and "Calibrated Position Error" 
TABLE I

DESIGN GOALS AND MEASURED RESULTS.

\begin{tabular}{|c|c|c|c|}
\hline System Metric & State of the art & Goal & Results \\
\hline Navigation accuracy for $1 / 2$ hour walk $[\mathrm{m}]$ & 45 & $\leq 10$ & $4.30 \pm 3.15$ \\
\hline Zero-velocity sensing bias per step $[\mathrm{mm} / \mathrm{s}]$ & 28 & $\leq 4$ & 4 (imaging limited) \\
\hline Form factor for velocity sensor alone $[\mathrm{cc}]$ & 125 & $\leq 10$ & 5 \\
\hline Power consumption of the velocity sensor $[\mathrm{mW}]$ & 2000 & $\leq 300$ & 20 \\
\hline Number of GRSC sensor elements & 10 & $\geq 10$ (COTS) & 24 (COTS) \\
\hline
\end{tabular}

State of the art navigation accuracy is projected from a shorter walk that was reported in [9].

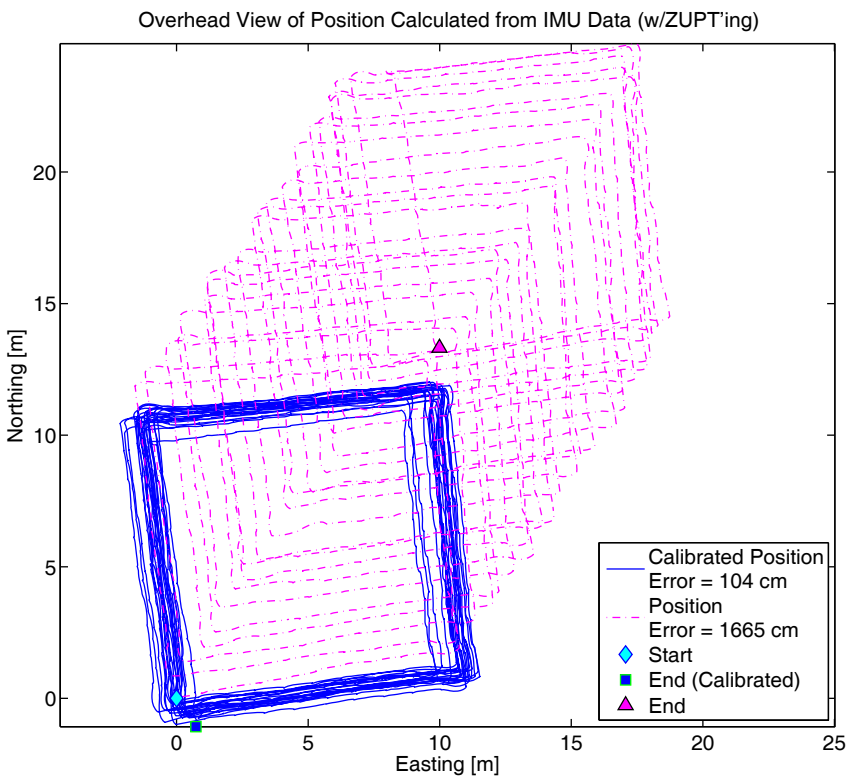

Fig. 13. $1 / 2$ hour square loop walk results. Position is calculated by integrating the measured acceleration, $a_{m}$, with ZUPT'ing, and Calibrated Position is calculated from integrated actual acceleration, $a_{a}$, with ZUPT'ing followed by the calibration. Loop closing error is $1.04 \mathrm{~m}(0.098 \%)$.

is calculated from integrated actual acceleration, $a_{a}$, with ZUPT'ing followed by the calibration. Figure 13 shows a walk experiment where the user followed the square loop for the whole experiment. Loop closing error for this experiment is $1.04 \mathrm{~m}(0.098 \%)$. This result shows the effectiveness of the calibration process that was performed. Figure 14 shows a calibration walk followed by a random walk experiment in the field. Loop closing error for this experiment is $1.45 \mathrm{~m}$ $(0.078 \%)$.

The average path length walked during $1 / 2$ hour walks was $1215 \mathrm{~m}$ (this is in addition to an average $235 \mathrm{~m}$ calibration walks). We have conducted 6 walk experiments, and the loop-closing errors has a mean of $4.30 \pm 3.15 \mathrm{~m}$ (mean \pm standard deviation), which makes the average relative error $0.35 \%$

\section{CONCluding Remarks And Future Work}

We have developed a personnel micronavigation system that uses inertial measurements from an IMU and zero velocity measurements from a GRSC. Our design goals,

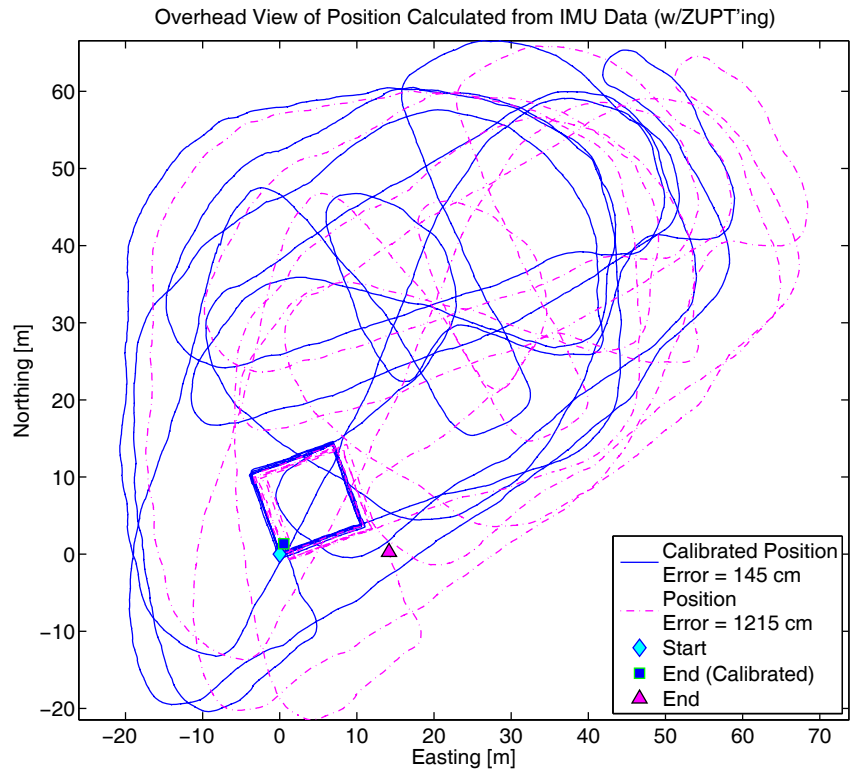

Fig. 14. $1 / 2$ hour random walk results. Position is calculated by integrating the measured acceleration, $a_{m}$, with ZUPT'ing, and Calibrated Position is calculated from integrated actual acceleration, $a_{a}$, with ZUPT'ing followed by the calibration. Loop closing error is $1.45 \mathrm{~m}(0.078 \%)$.

specifications from state of the art, and results from the developed system are given in Table I. All of the design goals were achieved. Only the zero-velocity sensing bias goal was reached indirectly. That is due to the placement of the pressure sensor array in the shoe. The closer the sensor is to the ground, the better the detection of zero-velocity regions get. Although the pressure sensor array in our setup was placed under the insole of the shoe, we were able to observe the zero-velocity points effectively using pressure contour velocities.

In our future work, would like to use a higher density pressure sensor array. As the number of the pressure sensor elements increase, the resolution of the pressure contours increase, and with that the minimum detectable zero velocity becomes closer to zero. This would improve the performance of ZUPT'ing. We also would like to use a differential global positioning system (DGPS) to validate our random walk results throughout the trajectory, where in this study only loop-closing errors were used to verify the system accuracy. Our straight-walk and around-the-square-loop experiments 
show that we did not have any other form of error that would not be shown by loop-closure check, but would be visible in DGPS data.

\section{REFERENCES}

[1] J. Kim, J.-G. Lee, G.-I. Jee, and T. K. Sung, "Compensation of gyroscope errors and gps/dr integration," in Proc. IEEE Position Location and Navigation Symposium, April 1996, pp. 464-470.

[2] Z. Zhou, X. Chen, and T. Zhang, "The principle of non-sensor dead reckoning," in Proc. IEEE Geoscience and Remote Sensing Symposium, vol. 7, July 2005, pp. 4693-4696.

[3] W.-W. Kao, "Integration of gps and dead reckoning navigation systems," in Proc. IEEE Vehicle Navigation and Information Systems Conference, vol. 2, October 1991, pp. 635-643.

[4] E. S. Sazonov, T. Bunpus, S. Zeigler, and S. Marocco, "Classification of plantar pressure and heel acceleration patterns using neural networks," in Proc. IEEE Neural Networks Conference, vol. 5, JulyAugust 2005, pp. 3007-3010.

[5] M. Lord and R. Hosein, "Pressure distribution by molded inserts in diabetic footwear: a pilot study," J. Rehab. Res. Dev., vol. 31, no. 3, pp. 214-221, 1994.

[6] C. Randell, C. Djiallis, and H. Muller, "Personal position measurement using dead reckoning," in Proc. IEEE International Symposium on Wearable Computers, October 2005, pp. 166-173.

[7] L. Ojeda and J. Borenstein, "Non-gps navigation for emergency responders," in International Joint Topical Meeting: Sharing Solutions for Emergencies and Hazardous Environments, Salt Lake City, Utah, USA, February 2006.

[8] _ "Non-gps navigation with the personal dead reckoning system," in SPIE Defense and Security Conference, Unmanned Systems Technology IX, Orlando, Florida, USA, April 2007, pp. 1-11.

[9] —_, "Non-gps navigation for security personnel and emergency responders," The Journal of Navigation, vol. 60, no. 3, pp. 391-407, September 2007.

[10] J. Borenstein, L. Ojeda, and S. Kwanmuang, "Heuristic reduction of gyro drift for personnel tracking systems," The Journal of Navigation, vol. 62, no. 1, pp. 41-58, January 2009.

[11] J. Cheung, M. Zhang, A. Leung, and Y. Fan, "Three-dimensional finite element analysis of the foot during standing-a material sensitivity study," Journal of Biomechanics, vol. 38, pp. 1045-1054, 2005.

[12] M. Trew and T. Everett, Human movement: an introductory text, 4th ed. Elsevier Health Sciences, 2001.

[13] H. Lanshammar and L. Strandberg, "Horizontal floor reaction forces and heel movements during the initial stance phase," Biomechanics VIII-B, pp. 1123-1128, 1982.
[14] T. Judd, "A personal dead reckoning module," in Proc. Inst. of Navigation Global Positioning System Conf., Kansas City, Missouri, USA, September 1997.

[15] M. Kourogi and T. Kurata, "Personal positioning based on walking locomotion analysis with self-contained sensors and a wearable camera," in Proc. 2nd IEEE and ACM International Symposium on Mixed and Augmented Reality (ISMAR), October 2003, pp. 103-112.

[16] R. Jirawimut, P. Ptasjnski, V. Garaj, F. Cecelja, and W. Balachandran, "A method for dead reckoning parameter correction in pedestrian navigation system," in Proceedings of the IEEE Instrumentation and Measurement Technology Conference, Budapest, Hungary, May 2001, pp. 1554-1558.

[17] J. Elwell, "Inertial navigation for the urban warrior," in Proc. SPIE Int. Society for Optical Engineering, vol. 3709, 1999, pp. 196-204.

[18] R. Stirling, J. Collin, K. Fyfe, and G. Lachapelle, "An innovative shoemounted pedestrian navigation system," in Proc. European Navigation Conf. (GNSS). Australian Inst. of Navigation, January 2003.

[19] S. Y. Cho and C. G. Park, "MEMS based pedestrian navigation system," The Journal of Navigation, vol. 59, pp. 135-153, 2006.

[20] J. Saarinen, J. Suomela, S. Heikkila, M. Elomaa, and A. Halme, "Personal navigation system," in Proc. IEEE/RSJ International Conference on Intelligent Robots and Systems (IROS), vol. 1, 2004, pp. 212-217.

[21] T. Brand and R. Phillips, "Foot-to-foot range measurement as an aid to personal navigation," in 59th Institute of Navigation Annual Meeting, Albuquerque, New Mexico, USA, 2003.

[22] R. Feliz, E. Zalama, and J. G. Garcia-Bermejo, "Pedestrian tracking using inertial sensors," Journal of Physical Agents, vol. 3, no. 1, pp. 35-42, January 2009.

[23] E. Foxlin, "Pedestrian tracking with shoe-mounted inertial sensors," IEEE Computer Graphics and Applications, vol. 25, no. 6, pp. 38-46, Nov-Dec 2005.

[24] Eagle Digital RealTime System, Motion Analysis Corporation, Santa Rosa, CA 95403, USA, 2009. [Online]. Available: http://www.motionanalysis.com/html/movement/eagle.html

[25] Pedar system, pressure insole size $X W$, Novel Electronics Incorporated, Munich, Germany, 2009. [Online]. Available: http://www.novelusa.com/index.php?fuseaction=systems.pedar

[26] Product Manual for use with InterSense InertiaCube3 and the InterSense InertiaCube Processor, 2nd ed., InterSense, Inc, Bedford, MA 01730, USA, 2007. [Online]. Available: www.intersense.com/products/downloads/InertiaCube3\%5FManual.pdf

[27] G. F. Franklin, J. D. Powell, and M. Workman, Digital Control of Dynamic Systems, 3rd ed. Addison Wesley Longman, 1998. 